\title{
Erratum to: Solving nonlinear problems by Ostrowski-Chun type parametric families
}

\author{
Alicia Cordero • Javier G. Maimó • \\ Juan R. Torregrosa • María P. Vassileva
}

Published online: 17 February 2015

(C) Springer International Publishing Switzerland 2015

\section{Erratum to: J Math Chem (2015) 53:430-449 DOI 10.1007/s10910-014-0432-z}

The following errors were inadvertently overlooked in the original publication, and it has been corrected with this erratum.

Page 8. The first line of the formula (13) says: " $y^{(k+1)}=x^{(k)}-\alpha\left[F^{\prime}\left(x^{(k)}\right)\right]^{-1}$ $F\left(x^{(k)}\right)$ ", replace by $y^{(k)}=x^{(k)}-\alpha\left[F^{\prime}\left(x^{(k)}\right)\right]^{-1} F\left(x^{(k)}\right)$, and the last two lines of same formula are repetitions: delete.

Page 9. Line 13 says: "where $e_{k}=x^{(k)}-\xi$ and $C_{q}=\left(\frac{1}{q !}\right)\left[f^{\prime}(\xi)\right]^{-1} F^{(q)}(\xi), q \geq$ 2." Replace by $e_{k}=x^{(k)}-\xi$ and $C_{q}=\left(\frac{1}{q !}\right)\left[F^{\prime}(\xi)\right]^{-1} F^{(q)}(\xi), q \geq 2$.

Page 10. The line 20 says: " $e_{k+1}=H_{2}^{\prime} e_{k}^{2}+H_{3}^{\prime} e_{k}^{2}+H_{4}^{\prime} e_{k}^{2}+\mathcal{O}\left[e_{k}^{5}\right]$ ”. Replace by $e_{k+1}=H_{2}^{\prime} e_{k}^{2}+H_{3}^{\prime} e_{k}^{3}+H_{4}^{\prime} e_{k}^{4}+\mathcal{O}\left[e_{k}^{5}\right]$.

The online version of the original article can be found under doi:10.1007/s10910-014-0432-z.

A. Cordero · J. R. Torregrosa

Instituto de Matemática Multidisciplinar, Universitat Politécnica de Valencia, Valencia, Spain e-mail: acordero@mat.upv.es

\section{J. R. Torregrosa}

e-mail: jrtorre@mat.upv.es

\section{J. G. Maimó · M. P. Vassileva (凶)}

Instituto Tecnológico de Santo Domingo (INTEC), av. Los Próceres, Galá, Santo Domingo, República Dominicana

e-mail: maria.penkova@intec.edu.do

J. G. Maimó

e-mail: javier.garcia@intec.edu.do 
Page 10. The line 21 says: "where $H_{1}^{\prime}=\frac{1}{a_{1}}\left(1+a_{1}\left(b_{1}-1\right)\right) C_{2}$. Replace by: where $H_{2}^{\prime}=\frac{1}{a_{1}}\left(1+a_{1}\left(b_{1}-1\right)\right) C_{2}$.

Page 11. The line 6 says: " $G\left(x^{(k)}, y^{(k)}\right)=\frac{1}{a_{1}}\left[\left(1+a_{1} b_{2}-2 a_{1}\right) I-a_{1}\left(b_{2}-\right.\right.$ 2) $\left.\left[F^{\prime}\left(x^{(k)}\right)\right]^{-1}\left[x^{(k)}, y^{(k)} ; F\right]\right]^{-1}$ ". Replace by: $G\left(x^{(k)}, y^{(k)}\right)=\frac{1}{a_{1}}\left[\left(1+a_{1} b_{2}-2 a_{1}\right) I-\right.$ $\left.a_{1}^{2}\left(b_{2}-2\right)\left[F^{\prime}\left(x^{(k)}\right)\right]^{-1}\left[x^{(k)}, y^{(k)} ; F\right]\right]^{-1}$.

Page 11. The line 19 says: " $x^{(k+1)}=y^{(k)}-\left(I-2\left[F^{\prime}\left(x^{(k)}\right)\right]^{-1}\left[x^{(k)}, y^{(k)} ; F\right]\right)$ $\left[F^{\prime}\left(x^{(k)}\right)\right]^{-1} F\left(y^{(k)}\right)$ ". Replace by $x^{(k+1)}=y^{(k)}-\left(3 I-2\left[F^{\prime}\left(x^{(k)}\right)\right]^{-1}\left[x^{(k)}, y^{(k)} ; F\right]\right)$ $\left[F^{\prime}\left(x^{(k)}\right)\right]^{-1} F\left(y^{(k)}\right)$.

Page 11. The line 25 says: " $x(k+1)=y^{(k)}-\frac{1}{a_{1}}\left[\left(1-a_{1}\right) I+a_{1}\left[F^{\prime}\left(x^{(k)}\right)\right]^{-1}\left[x^{(k)}\right.\right.$, $\left.\left.y^{(k)} ; F\right]\right]^{-1}$,. Replace by $x^{(k+1)}=y^{(k)}-\frac{1}{a_{1}}\left[\left(1-a_{1}\right) I+a_{1}^{2}\left[F^{\prime}\left(x^{(k)}\right)\right]^{-1}\left[x^{(k)}, y^{(k)} ; F\right]\right]^{-1}$

Page 11. The line 26 says: " $F\left(x^{(k)}\right)+\frac{1}{a_{1}}\left[\left(2 a_{1}-1\right) I-\left[F^{\prime}\left(x^{(k)}\right)\right]^{-1}\left[x^{(k)}, y^{(k)} ; F\right]\right]$ $\left[F^{\prime}\left(x^{(k)}\right)\right]^{-1} F\left(y^{(k)}\right)$ ". Replace by $F\left(x^{(k)}\right)+\frac{1}{a_{1}}\left[\left(2 a_{1}-1\right) I-\left[F^{\prime}\left(x^{(k)}\right)\right]^{-1}\left[x^{(k)}, y^{(k)}\right.\right.$; $F]]\left[F^{\prime}\left(x^{(k)}\right)\right]^{-1} F\left(y^{(k)}\right)$.

Page 15. The line 1 says: "Table 3 Test functions and results for nonlinear systems, $F_{1}$ and $F_{2}$ ". Replace by: Table 3 Test functions and results for nonlinear functions, $F_{1}$ and $F_{2}$.

Page 15. The line 3 says: " $F_{1}\left(x_{1}, x_{2}\right)=\left(\exp x_{1} \exp x_{2}+x_{1} \cos x_{2}, x_{1}+x_{2}-\right.$ $1 x^{(0)}=(3,-2)$ and $\xi_{1} \approx$ ". Replace by $F_{1}\left(x_{1}, x_{2}\right)=\left(\exp x_{1} \exp x_{2}+x_{1} \cos x_{2}, x_{1}+\right.$ $\left.x_{2}-1\right) x^{(0)}=(3,-2)$ and $\xi_{1} \approx$.

Page 15. The line 4 says: " $3.4675009642402, \xi_{2} \approx-2.4675009642402$ ". Replace by: $3.4706309600316, \xi_{2} \approx-2.4706309600316$.

Page 16. The line 1 says: "Table 4 Test functions and results for nonlinear systems, $F_{3}$ and $F_{4}$ ". Replace by: Table 3 Test functions and results for nonlinear functions, $F_{3}$ and $F_{4}$.

Page 16. The line 4 says: “- $0.8452567390376772 ; \xi_{2} \approx-0.7481414932526368$ ”. Replace by: $-0.8452567390376772, \xi_{2} \approx 0.7481414932526368$. 